\title{
Effective DBHF Method for Asymmetric Nuclear Matter and Finite Nuclei
}

\author{
Zhong-yu Ma* and Ling Liu \\ China Institute of Atomic Energy, Beijing 102413
}

(May 5, 2002)

\begin{abstract}
A new decomposition of the Dirac structure of nucleon self-energies in the Dirac Brueckner-Hartree-Fock (DBHF) approach is adopted to investigate the equation of state for asymmetric nuclear matter. The effective coupling constants of $\sigma, \omega, \delta$ and $\rho$ mesons with a density dependence in the relativistic mean field approach are deduced by reproducing the nucleon self-energy resulting from the DBHF at each density for symmetric and asymmetric nuclear matter. With these couplings the properties of finite nuclei are investigated. The agreement of charge radii and binding energies of finite nuclei with the experimental data are improved simultaneously in comparison with the projection method. It seems that the properties of finite nuclei are sensitive to the scheme used for the DBHF self-energy extraction. We may conclude that the properties of the asymmetric nuclear matter and finite nuclei could be well described by the new decomposition approach of the G matrix.
\end{abstract}

Typeset using REVTEX

\footnotetext{
*Also Center of Nuclear Theoretical Physics, National Laboratory of Heavy Ion Accelerator and China Institute of Theoretical Physics.
} 


\section{INTRODUCTION}

The Dirac Brueckner Hartree-Fock (DBHF) approximation is a microscopic approach to study the saturation property of infinite nuclear matter, which is based on realistic nucleonnucleon interaction and contains features of the relativistic theory $1 \mathbb{1} 4$. The presently popular relativistic mean field (RMF) theory [5,6] is considered to be a phenomenological method in comparison with the DBHF approach. There are at least six free parameters in the RMF calculations, such as NL1 [7], NL3 [8] and NL-SH [9]. These parameters are adjusted by reproducing nuclear matter saturation properties and bulk properties of large number of stable nuclei, such as binding energies and charge radii. Consistent results with various parameter sets can be achieved for stable nuclei. However, discrepancies occur when the calculation is extended to nuclei in drip lines or super-heavy nuclei. The DBHF method adopts the realistic nucleon-nucleon (NN) interaction which is fitted to the NN scattering phase shifts and deuteron properties. It takes the nucleon in-medium short-range correlation effect into account by performing a complete summation of two-body ladder diagrams. Therefore the DBHF is generally accepted as one of the most reliable and feasible microscopic method for the description of effective interactions in the nuclear medium. A break-through with the DBHF theory was that it reproduces empirical saturation properties

of nuclear matter successfully [2], and presents a reasonable nuclear equation of state (EOS). This kind of EOS, obtained directly from a microscopic theory, contains more physical meanings than phenomenological models, especially for the investigation of the high-density and high-temperature behavior in the astrophysics as well as the collision of energetic heavy ions.

Attempts have been made to calculate the finite nuclei with effective meson-nucleon interactions, which are deduced from the DBHF self-energy. One of the most successful approaches in this direction is the so called relativistic density dependent Hartree [10] or Hartree-Fock [11,12, which could reasonably describe the properties of stable nuclei. In this approach the coupling constants of isoscalar $\sigma$ and $\omega$ mesons are adjusted at each density by 
reproducing the nucleon self-energies resulting from the DBHF. In this method, the Dirac structure of the self-energy in the DBHF G matrix was extracted from the momentum dependence of the single particle energy, where the momentum dependence of scalar and vector potentials was neglected [2]. This simple method encountered an obstacle in extracting the effective coupling constants of isovector mesons when it extends to asymmetric nuclear matter [13]. It was discussed that such approach may yield an isospin dependence of the effective interaction with a wrong sign [14]. Another scheme to extract the Dirac energy is the so called projection method 15,16]. Some ambiguities with pseudo-scalar and pseudo-vector couplings may be observed in the projection method due to the fact that positive energy Dirac spinors are used in the DBHF calculation only. In order to overcome these drawbacks, recently Schiller and Muether [17,18] suggested a new decomposition approach of the DBHF G-matrix in extracting the Dirac structure of the nucleon self energy. They separated the G-matrix into the bare $\mathrm{NN}$ interaction $\mathrm{V}$ and correlation $\Delta G$. The projection method is applied only on the correlation term $\Delta G$, which is parameterized by four pseudo-mesons. The ambiguities in the projection method are eliminated and a satisfactory description for the symmetric nuclear matter is achieved in this scheme.

In this paper we shall study the properties of asymmetric nuclear matter as well as neutron matter with the new decomposition of the Dirac structure in the DBHF G matrix. The main purpose of this paper is to extract an effective NN interaction with an isospin dependence from the DBHF nucleon self-energy in the symmetric and asymmetric nuclear matter. The effective nucleon-meson coupling constants with a density dependence contain the DBHF short-range correlations and information of the isospin structure. The properties of finite nuclei are investigated with these effective coupling constants.

This paper is arranged as the follows. In Sec.II the new decomposition of the Dirac structure of the DBHF G matrix is presented. The properties of asymmetric nuclear matter are discussed in Sec.III. The effective NN interaction with density dependent nucleon-meson couplings is extracted from the DBHF G matrix, which is described in Sec.IV. Studies of finite nuclei with the effective NN interaction in the RMF are depicted in Sec.V. Finally we 
give a brief summary in Sec.VI.

\section{A NEW DECOMPOSITION OF DBHF G MATRIX}

In the new decomposition approach, the DBHF G matrix is split into two parts [17], the bare NN interaction $V$ and correlation term $\Delta G$ :

$$
G=V+\Delta G
$$

Usually the bare NN interaction $V$ in the DBHF calculation is taken as one boson exchange potential (OBEP), such as Bonn potentials. The OBEP of Bonn potentials is associated with three isoscalar mesons $(\sigma, \omega$ and $\eta)$ and three isovector mesons $(\delta, \rho$ and $\pi)$ with the following quantum numbers $\left(J^{\pi}, T\right): \sigma\left(0^{+}, 0\right), \omega\left(1^{-}, 0\right), \quad \eta\left(0^{-}, 0\right), \quad \delta\left(0^{+}, 1\right), \quad \rho\left(1^{-}, 1\right), \pi\left(0^{-}, 1\right)$. The Dirac structure of the OBEP is explicitly known and its contribution to the nucleon selfenergy can be calculated in the relativistic Hartree-Fock approach. Analyzing the correlation term $\Delta G$, the peculiar property of the dominating $\pi$-exchange contribution is removed, therefore the projection method can be applied to $\Delta G$ without ambiguities. Schiller and Muether [17, 18] observed that the inclusion of $\Delta G$ leads to a constant shift in the nucleon scalar and vector potentials in comparison with those calculated with only OBEP, which is almost independent of the nucleon momentum. Therefore, the correlation term $\Delta G$, can be well described in terms of an effective interaction with zero range. It is parameterized by four isoscalar and isovector effective mesons : $\left(\sigma^{\prime}, \omega^{\prime}, \delta^{\prime}\right.$ and $\left.\rho^{\prime}\right)$ with infinite masses, which are defined as pseudo-mesons. The ratios of coupling constants and corresponding masses for those psuedo-mesons are finite and of weak density dependence. In this way, the

contribution of the correlation effect $\Delta G$ to the self energy can also be calculated in the relativistic Hartree-Fock approach.

The self-energy of protons and neutrons with a momentum $k$ in nuclear matter can be decomposed into scalar and time-like and space-like vector components.

$$
\Sigma^{i}(k)=\Sigma_{s}^{i}(k)-\gamma^{0} \Sigma_{0}^{i}(k)+\gamma \cdot \mathbf{k} \Sigma_{v}^{i}(k)
$$


where $i$ denotes the proton and neutron. It is convenient to eliminate the space-like vector component $\Sigma_{v}^{i}$ and write the Dirac equation into a form which contains only scalar and time-like vector components in a usual way,

$$
\left\{\vec{\gamma} \cdot \vec{k}+\left[M+\tilde{\Sigma}_{s}^{i}(k)\right]-\tilde{\Sigma}_{0}^{i}(k) \gamma^{0}\right\} u_{i}(k)=\varepsilon_{i}(k) \gamma^{0} u_{i}(k)
$$

where

$$
\tilde{\Sigma}_{s}^{i}=\frac{\Sigma_{s}^{i}-M \Sigma_{v}^{i}}{1+\Sigma_{v}^{i}}, \quad \widetilde{\Sigma}_{0}^{i}=\frac{\Sigma_{s}^{i}-\varepsilon_{i} \Sigma_{v}^{i}}{1+\Sigma_{v}^{i}}
$$

We define the nucleon effective mass,

$$
M^{*}(k)=M+\tilde{\Sigma}_{s}^{i}(k)
$$

The single-particle energy takes the form,

$$
k_{i}^{0}=\varepsilon_{i}(k)=\sqrt{k^{2}+M_{i}^{*}(k)^{2}}-\widetilde{\Sigma}_{0}(k)=E^{*}(k)-\widetilde{\Sigma}_{0}(k) .
$$

The Dirac structure of the nucleon self-energy in nuclear matter in the DBHF approach can be calculated with the OBEP $V$ and $\Delta G$, which is parameterized with psuedo-mesons.

$$
\Sigma(k)=\sum_{\alpha} \int \frac{d^{4} q}{(2 \pi)^{4}}\left\{\Gamma_{\alpha}^{a} D_{\alpha}^{a b}(0) \operatorname{Tr}\left[i \mathcal{G}(q) \Gamma_{\alpha}^{b}\right]-\Gamma_{\alpha}^{a}[i \mathcal{G}(q)] D_{\alpha}^{a b}(k-q) \Gamma_{\alpha}^{b}\right\}
$$

where the first and second terms are direct and exchange contributions, respectively. The index $\alpha$ refers to mesons and $a$, and $b$ refer to isospin components. $\mathcal{G}(q)$ is the single particle Green's function,

$$
\mathcal{G}(k)=\left(\gamma^{\mu} k_{\mu}+M^{*}\right)\left(\frac{1}{k_{\mu}^{2}-M^{* 2}+i \eta}+\frac{i \pi}{\varepsilon_{k}} \delta\left(k_{0}-\varepsilon_{k}\right) \Theta\left(k_{F}-|\boldsymbol{k}|\right)\right)
$$

$D_{\alpha}^{a b}(q)$ are meson propagators, which have the following forms for scalar and vector mesons, respectively.

$$
\begin{aligned}
& D_{s}^{a b}(k)=\frac{1}{k_{\mu}^{2}-m_{s}^{2}+i \eta} T^{a b} \\
& D_{v}^{a b}(k)=\frac{-g_{\mu \nu}+k_{\mu} k_{\nu} / m_{v}^{2}}{k_{\mu}^{2}-m_{v}^{2}+i \eta} T^{a b}
\end{aligned}
$$


where $T^{a b}$ is the isospin operator, $\mathbb{1}$ for isoscalar and $\delta_{a b}$ for isovector mesons, respectively. The vertex operators $\Gamma_{\alpha}^{a}$ for scalar, vector and pseudoscalar mesons are expressed as the follows. The vector mesons have both vector and tensor couplings and pseudo-vector couplings for pseudoscalar mesons are adopted.

$$
\Gamma_{\alpha}^{a}=t^{a}\left\{\begin{array}{cc}
i g_{s} & \text { for scalar mesons } \\
i\left(g_{v} \gamma^{\mu}+\frac{f_{v}}{2 M} \partial_{\nu} \sigma^{\nu \mu}\right) & \text { for vector mesons } \\
i \frac{f_{p s}}{m_{p s}} \gamma^{5} \gamma^{\mu} \partial_{\mu} & \text { for pseudo-scalar mesons }
\end{array}\right.
$$

The isospin operator $t^{a}$ equals $\mathbb{1}$ for isoscalar mesons and $\tau_{a}$ for isovector mesons.

\section{ASYMMETRIC NUCLEAR MATTER}

For asymmetric nuclear matter one defines the asymmetry parameter, $\beta=\left(\rho^{n}-\rho^{p}\right) /\left(\rho^{p}+\right.$ $\left.\rho^{n}\right)$, where $\rho^{p}$ and $\rho^{n}$ denote the proton and neutron density, respectively. It implies that $\beta=0$ for the symmetric nuclear matter and $\beta=1$ for the pure neutron matter. The nucleon self-energies for protons and neutrons have to be calculated individually.

The scalar and vector potentials in symmetric nuclear and neutron matter with the $G$ matrix have been investigated in Ref. [17,19]. Significant differences in scalar and vector potentials were observed in comparison with those obtained by using a simple method, where the nucleon self-energy was deduced from the single-particle energy, although the EOS produced by both methods were very similar. In this paper we present the study of the EOS for asymmetric nuclear matters. The Bonn A potential is chosen as the bare NN potential in the DBHF calculation and the coupling constants of pseudo mesons are taken from Ref. [17]. The EOS for various asymmetric parameters are given in Fig.1. For the asymmetry parameter $\beta<0.8$, the energy per nucleon as a function of the density exhibits

a curve with a minimum, but the saturation point shifts to lower densities as the asymmetry parameter increases. However, for the extreme neutron-rich or neutron matter $(\beta=1)$ no minimum in the EOS is found. 
The EOS of nuclear and neutron matters are two extreme cases, which determine the density dependence of the asymmetry energy $a_{\text {asym }}(\rho)$. In Fig.2(a) bounding energies per nucleon $E / A(\rho, \beta)$ at various densities are plotted as a function of $\beta^{2}$. Straight lines are drawn from the linear fit of the first three points at small asymmetric parameters $\beta$. It is found that those lines go through most numerical points and only small deviations are observed at $\beta=1$. It illustrates that the empirical parabolic law is fulfilled, which is consistent with the finding in the non-relativistic approach [20]. Therefore the asymmetry energy $a_{\text {asym }}(\rho)$ could be expressed as $E / A(\rho, \beta=1)-E / A(\rho, \beta=0)$. The density dependence of the asymmetric energy in nuclear matter is shown in Fig.2(b). The dashed curve is obtained in a non-relativistic BHF calculation taken from Ref. 21]. Both of them give similar asymmetric energy around the saturation density and below, but diverge at large densities.

\section{EFFECTIVE NN INTERACTION}

From scalar and vector self-energies in asymmetric nuclear matter calculated with the effective $G$ matrix we could extract the density dependence of effective coupling constants of isoscalar and isovector mesons. Following Brockman's method [10] we impose a condition that the nucleon self-energy at each nuclear density and asymmetric parameter obtained in the DBHF is reproduced by the effective $\mathrm{NN}$ interaction in the RMF calculation. The momentum dependence of the self-energy $\widetilde{\Sigma}_{s}^{i}$ and $\widetilde{\Sigma}_{0}^{i}$ is rather weak, so it is reasonable to take their mean values $U_{s}^{i}$ and $U_{0}^{i}$ from $k=0$ to $k=k_{F}^{i}$. The effective coupling constants of $\sigma, \omega, \delta$ and $\rho$ mesons can be determined as the follows,

$$
\begin{aligned}
\left(\frac{g_{\sigma}\left(\rho_{B}\right)}{m_{\sigma}}\right)^{2} & =-\frac{1}{2} \frac{U_{s}^{p}\left(\rho_{B}\right)+U_{s}^{n}\left(\rho_{B}\right)}{\rho_{s}^{p}+\rho_{s}^{n}} \\
\left(\frac{g_{\delta}\left(\rho_{B}\right)}{m_{\delta}}\right)^{2} & =-\frac{1}{2} \frac{U_{s}^{p}\left(\rho_{B}\right)-U_{0}^{n}\left(\rho_{B}\right)}{\rho_{s}^{p}-\rho_{s}^{n}} \\
\left(\frac{g_{\omega}\left(\rho_{B}\right)}{m_{\omega}}\right)^{2} & =-\frac{1}{2} \frac{U_{0}^{p}\left(\rho_{B}\right)+U_{0}^{n)}\left(\rho_{B}\right)}{\rho^{p}+\rho^{n}} \\
\left(\frac{g_{\rho}\left(\rho_{B}\right)}{m_{\rho}}\right)^{2} & =-\frac{1}{2} \frac{U_{0}^{p}\left(\rho_{B}\right)-U_{0}^{n}\left(\rho_{B}\right)}{\rho^{p}-\rho^{n}}
\end{aligned}
$$


where $\rho_{B}=\rho^{p}+\rho^{n}$. In general, the ratios of $g / m$ could be functions of the scalar or vector density and both the coupling constant and mass are of density dependence. In this paper we assume that only coupling constants depend on the vector density $\rho_{B}$ and masses of mesons remain constant. The coupling constants of $\sigma$ and $\omega$ mesons are taken from their values at $\beta=0$ in order to reproduce the DBHF results in nuclear matter. For the isospin dependence, we calculate the coupling constants for isovector mesons in various asymmetric nuclear matters with $\beta=0.2,0.4$, and 0.6 , respectively, and then take their averaged values.

The coupling constants as a function of the density are displayed in Fig.3. The solid circles are the results obtained from the nucleon self-energies in Eq.11. Due to a phase transition at very low density $k_{F}<1.0 \mathrm{fm}^{-1}$, the DBHF calculation can not be applied. Therefore, the extrapolation of coupling constants at low densities has to be performed when one tries to study the properties of finite nuclei. To give a guide line of the extrapolation by fittings, the Hartree-Fock calculation with the $\mathrm{G}$ matrix at $k_{F}=1.0 \mathrm{fm}^{-1}$ is carried out, which are plotted by open circles. The solid curves are drawn by a fitting with exponential forms as : $g_{\alpha}^{2}\left(\rho_{B}\right)=a_{0}+a_{1} \exp \left(-\rho_{B} / a_{2}\right)$ for $\alpha=\sigma, \omega, \rho$ and a polynomial form: $g_{\delta}^{2}\left(\rho_{B}\right)=$ $a_{0}+a_{1} \rho_{B}+a_{2} \rho_{B}^{2}$ for $\delta$ meson. The parameters of fitting are listed in Table. 1 and marked by Fit.A.

The RMF calculation by the effective nucleon-meson couplings reproduces the DBHF nucleon self-energies, but not the EOS. The deviation of the binding energy at the saturation point is within $2 \%$ and increases at high densities. We also perform another extraction procedure for effective couplings by imposing the condition of reproducing the DBHF scalar potential and binding energy per nucleon at each density for symmetric and asymmetric nuclear matters. In this way, the EOS and asymmetry energy in the DBHF calculation are exactly reproduced in the RMF calculation with the new set of effective nucleon-meson couplings. The parameters of this kind of fitting are also shown in Table. 1 and denoted by Fit.B.

In Fig.3 we plot the coupling constants obtained from Ref. [2, 10] and Ref. [22] for a comparison. The dashed curves are the results in Ref. [2,10], where the Dirac structure of 
nucleon self energies in the DBHF calculation is extracted from the momentum dependence of the single particle energy. These coupling constants are obtained through those scalar and vector self-energies at nuclear and neutron matter. It is found that the results are very different from ours, although the DBHF G matrix is obtained from the same OBEP of Bonn A in both methods. The dependence of coupling constants on the density in Ref. 13] is extremely strong. Furthermore, the absolute values of isovector mesons $\left(g_{\delta}, g_{\rho}\right)$ are very large and have the same magnitude as those for isoscalar mesons $\left(g_{\sigma}, g_{\omega}\right)$. It should be noted that the square values of coupling constants for isovector mesons turn out to be negative in that analysis, which means that the coupling constants themselves would be imaginary. Those are not consistent with our common understanding. It can be seen from those solid curves that our coupling constants exhibit a moderate density dependence, and the strengths for isovector mesons are much weaker than those of isoscalar mesons. The dotted curves in Fig.3 are the results taken from Ref. [22], where the DBHF self-energy is extracted in terms of a projection method. Those couplings are taken from their average values obtained in asymmetry nuclear matters with $\beta=0.2,0.4$, and 0.6 . It is also seen that the coupling constants from the projection method is stronger than our results, especially for isovector mesons.

\section{FINITE NUCLEI}

We study the properties of some doubly magic and semi-magic nuclei, such as ${ }^{16} \mathrm{O}$, ${ }^{40} \mathrm{Ca},{ }^{48} \mathrm{Ca},{ }^{90} \mathrm{Zr},{ }^{208} \mathrm{~Pb},{ }^{48} \mathrm{Ni},{ }^{56} \mathrm{Ni},{ }^{68} \mathrm{Ni},{ }^{100} \mathrm{Sn}$, and ${ }^{132} \mathrm{Sn}$ in the RMF with the effective NN interaction. The rearrangement terms due to the density dependence in the effective nucleonmeson couplings are included in the calculation. The relative deviations of charge radii and binding energies $\left(x_{C a l}-x_{E x p}\right) / x_{E x p}$. are displayed in Figs.4(a) and 4(b), respectively. The solid (open) circles are our results calculated with the effective nucleon-meson couplings of Fit.A (Fit.B). The calculated charge radii are improved in the case of Fit.B, but the resulting binding energies become smaller in comparison with the experiment data. In 
a general speaking, an increase in the charge radius produced by one set of parameters would result in a decrease of the value of the binding energy. A better way to estimate a scheme is to investigate whether the calculated binding energies and charge radii could both be improved. In Ref. [22], they adopted a momentum correction in the extraction of couplings from the DBHF self energies in a projection method. In this way additional parameters are introduced, which are adjusted to the nuclear matter or finite nuclei. With the momentum correction they can improve the binding energy and charge radii at the same time. The upper and lower triangles are the results in Ref. [22] corresponding to the cases adjusted to the nuclear matter and finite nuclei, respectively. In our calculation we do not introduce additional parameters, except for the extrapolation at low densities, which are slightly adjusted by the properties of finite nuclei. Our results are improved simultaneously in binding energies and charge radii in comparison with those from Ref. [22], although the G matrix in Ref. [22 have been calculated from the Groningen parameterization. Actually the saturation properties in nuclear matter from the DBHF calculation with those two free NN interactions : Bonn $\mathrm{A}$ and Groningen interaction are very similar, which are $E / A=$ $-15.6 \mathrm{MeV}$ at $\rho_{B 0}=0.185 \mathrm{fm}^{-3}$ and $E / A=-15.5 \mathrm{MeV}$ at $\rho_{B 0}=0.182 \mathrm{fm}^{-3}$, respectively. However, both of binding energies and charge radii of our results are better than those in Ref. [22]. It seems that the properties of finite nuclei are sensitive to the scheme used for the DBHF self energy extraction.

The neutron and proton spin-orbit splittings $E_{S O}=E_{n, l, j=l-1 / 2}-E_{n, l, j=l+1 / 2}$ are examined in Fig. 5 for $1 p$ shell for ${ }^{16} \mathrm{O}$ and the $1 d$ shell for ${ }^{40} \mathrm{Ca},{ }^{48} \mathrm{Ca}$, and ${ }^{48} \mathrm{Ni}$. The solid and open circles represent the calculations with couplings of Fit.A and Fit.B in Table 1, respectively. Various experimental data 23,24] are also shown in Fig.5. Although the experimental values are not accurately established, our results are in good agreement with the experimental data except for ${ }^{48} \mathrm{Ca}$. The experimental data for ${ }^{40} \mathrm{Ca}$ and ${ }^{48} \mathrm{Ca}$ are divergent. The results show that the effective interaction deduced from the new Dirac structure decomposition of the G matrix could well describe the properties of finite nuclei in comparison with other methods. 


\section{A BRIEF SUMMARY}

In summary, we have applied the new decomposition of the Dirac structure of the nucleon self energy from the $\mathrm{G}$ matrix in the DBHF calculation to investigate properties of symmetric and asymmetric nuclear matter. The DBHF G matrix is composed of a OBEP and correlation term, which are parameterized by four pseudo-mesons with infinite masses. The EOS for asymmetric nuclear matter and the density dependence of the asymmetry energy are investigated with the DBHF G matrix. The effective NN interaction with a density dependence in the RMF is extracted from the DBHF G matrix. The effective coupling constants of isoscalar and isovector mesons incorporate the DBHF results and involve NN correlation effects. We performed calculations for finite nuclei using the effective nucleonmeson coupling constants. The bulk properties of finite nuclei are well described and the results are in good agreement with the experimental data. Both of binding energies and charge radii are improved simultaneously in comparison with the others [22], the deviations of which with the experimental data for some magic and semi-magic nuclei are within $5 \%$. It seems that the properties of finite nuclei are sensitive to the scheme used for the DBHF self energy extraction. Examinations of the spin-orbit split are also made. The results show good agreement with the experimental data available. Finally we may conclude that the splitting of the $\mathrm{G}$ matrix into a bare interaction and correlation term is a satisfactory way to extract the Dirac structure of the nucleon self energy in the DBHF. The properties of asymmetric nuclear matter and finite nuclei could be well described by the new decomposition approach of the G matrix. The application of this approach to the neutron star and investigation of effects of isovector mesons in exotic nuclei are the future interesting exploration.

\section{ACKNOWLEDGMENTS}

One of the authors MZY would like to thank Prof. H. Muether and Dr. E. Schiller for stimulating discussions and providing the parameters of the Dirac structure of the $G$ matrix. This work is supported by the National Natural Science Foundation of China under No. 10075080, 19835010 and Major State Basic Research Development Program under 
contract No. G20007740. 


\section{REFERENCES}

[1] R. Machleidt, Adv. Nucl. Phys., 19, 189 (1989).

[2] R. Brockmann and R. Machleidt, Phys. Lett. 149B, 283 (1984); Phys. Rev. C42, 1965 (1990); and private communication.

[3] F. de Jong, H. Lenske, Phys. Rev. C58, 890 (1998).

[4] H. Muether, R. Machleidt, and R.Brockmann, Phys. Rev. C42, 1981 (1990).

[5] B. D. Serot and J. D. Walecka, Adv. Nucl. Phys. 16, 1 (1986).

[6] P. Ring, Prog. Part. Nucl. Phys. 37, 193 (1996).

[7] P-G. Reinhard, M. Rufa, J. A. Maruhn, W. Greiner and J. Friedrich, Z. Phys., A323, $13(1986)$.

[8] G. A. Lalazissis, P. Ring, Phys. Rev., C55, 540 (1997).

[9] M. M. Sharma, M. A. Nagarajan, P. Ring, Phys. Lett., B312, 377 (1993).

[10] R. Brockmann and H. Toki,Phys. Rev. Lett., 68, 3408 (1992).

[11] Zhong-yu Ma, Hua-lin Shi,and Bao-qiu Chen, Phys. Rev., C50, 3170 (1994).

[12] Hua-lin Shi,Bao-qiu Chen,and Zhong-yu Ma, Phys. Rev., C52, 144 (1995).

[13] H. Shen, Y. Sugahara and H. Toki, Phys. Rev., C55, 1211 (1997).

[14] S. Ulrych and H. Muether, Phys. Rev., C56, 1788 (1997.

[15] F. Boersma and R. Malfliet, Phys. Rev., C49, 233 (1994).

[16] F. de Jong and H. Lenske, Phys. Rev. C57, 3099 (1998).

[17] E. Schiller and H. Muether, Eurphys.J., 11, 15 (2001).

[18] H. Muether and A. Polls, Prog. Part. Nucl. Phys., 45, 243 (2000). 
[19] Ling Liu and Zhong-yu Ma, Chin. Phys. Lett., 19, 190 (2002).

[20] Wei Zuo, I. Bombaci and U. Lombardo, Phys. Rev., C60, 024605 (1999).

[21] I. Bombaci and U. Lombardo, Phys. Rev., C44, 1892 (1991).

[22] F. Hofmann, C. M. Keil and H. Lenske, Phys. Rev., C64, 034314 (2001).

[23] C. Fucks, H. Lenske and H. H. Wolter, Phys. Rev., C52, 3043(1995).

[24] M. Lopez-Quelle, N. Van Giai, S. Marcos and L. N. Savushkin, Phys. Rev., C61, 064321 (2000). 


\section{TABLES}

\begin{tabular}{lclll}
\hline \hline & & $a_{0}$ & $a_{1}$ & $a_{2}$ \\
\hline Fit.A & $g_{\sigma}^{2}$ & 76.8226 & 48.4004 & 0.1332 \\
& $g_{\omega}^{2}$ & 97.7896 & 71.9705 & 0.2387 \\
& $g_{\rho}^{2}$ & 11.5862 & 21.3433 & 0.1385 \\
& $g_{\delta}^{2}$ & 5.4139 & 34.7149 & 15.4454 \\
\hline Fit.B & $g_{\sigma}^{2}$ & 77.7312 & 48.5497 & 0.1260 \\
& $g_{\omega}^{2}$ & 104.8553 & 69.0250 & 0.1955 \\
& $g_{\rho}^{2}$ & 10.8791 & 34.1492 & 0.0845 \\
& $g_{\delta}^{2}$ & 16.9648 & 52.2796 \\
\hline \hline
\end{tabular}

TABLE I. Parameters of effective coupling constants by fittings. The expressions of fittings are described in the text. Fit.A and Fi.B denote the fittings through the DBHF scalar and vector self-energies and by the DBHF scalar and binding energies, respectively. 


\section{FIGURE CAPTIONS}

FIG. 1. The equation of state for various asymmetry parameters from symmetric nuclear $\operatorname{matter}(\beta=0)$ to neutron matter $(\beta=1)$.

FIG. 2(a). The binding energy per nucleon $E / A(\rho, \beta)$ as a function of asymmetry parameter $\beta^{2}$. Fig. 2(b). The density dependence of the asymmetry energy. The dashed curve is the result of a non-relativistic Brueckner Hartree-Fock calculation [20].

FIG. 3. The density dependence of coupling constants for $\sigma, \omega, \delta$ and $\rho$ mesons. The solid circles are extracted from the DBHF self-energies in the present work. Open circles are obtained by extrapolation calculations with the G matrix at $k_{F}=1.0 \mathrm{fm}^{-1}$. The solid curves are by fittings with an exponential or polynomial form Fit.A in Table 1. The dashed lines are the results in Ref. [2,10], where the DBHF self-energies are determined from the single-particle energy. The dotted lines are obtained in Ref. [22] with a projection method. FIG. 4. The relative deviations of charge radii(a) and the binding energies(b) for some stable nuclei. The solid and open circles are our results with coupling constants Fit.A and Fit.B in Table.1, respectively. The upper and lower triangles are taken from Ref. [22] adjusted to the nuclear matter and finite nuclei, respectively. Lines are drawn to guide the eyes.

FIG. 5. The spin-orbit split for the proton (a) and neutron (b). The notations are the same as in Fig.4. The open lower triangles are also taken from Ref. [22], which are the same as the case with lower solid triangles except for that the Bonn A potential was adopted. The experimental data denoted by A [23] and B, C, D and E are taken from Ref. [24] and references cited therein. 


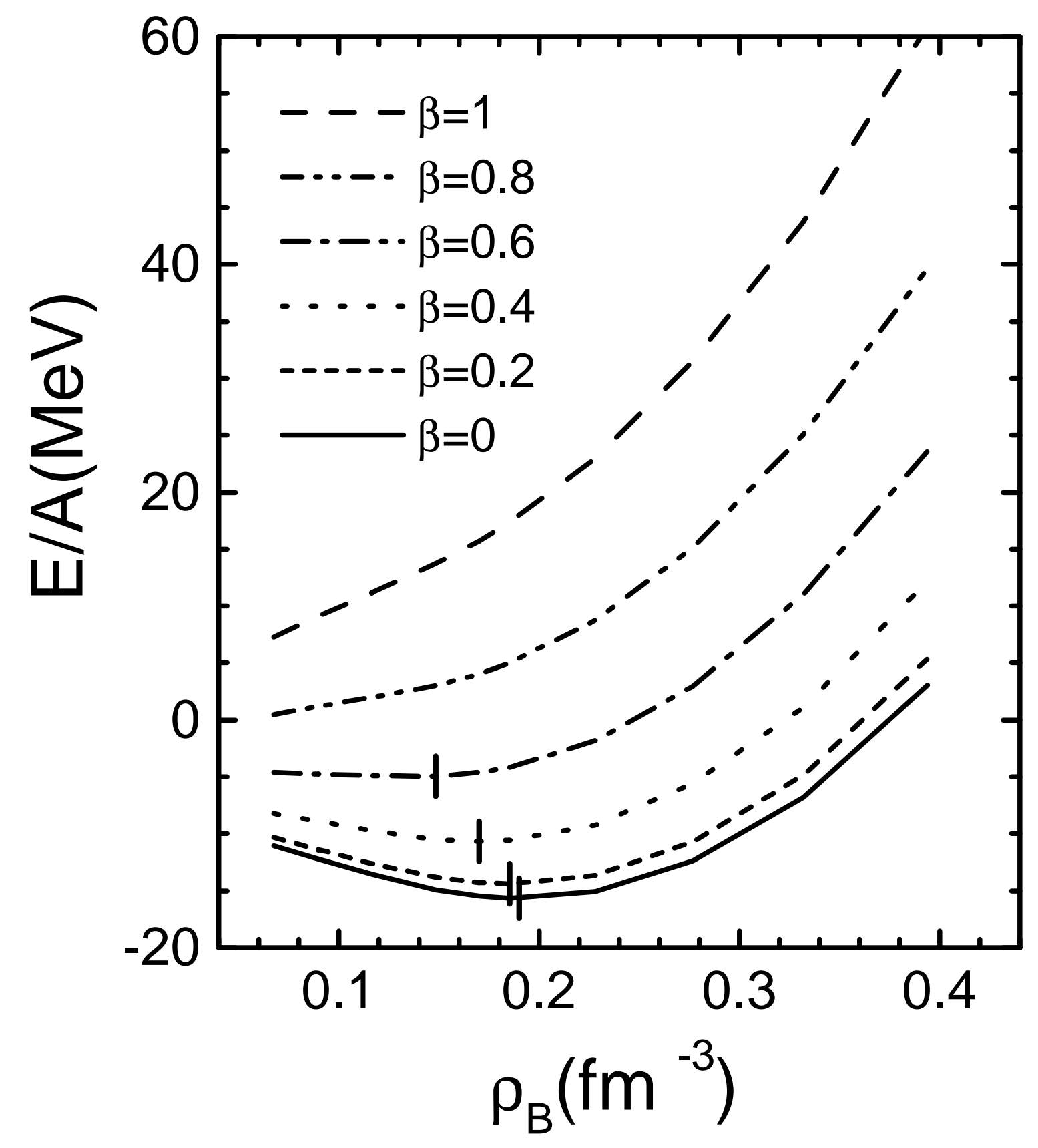




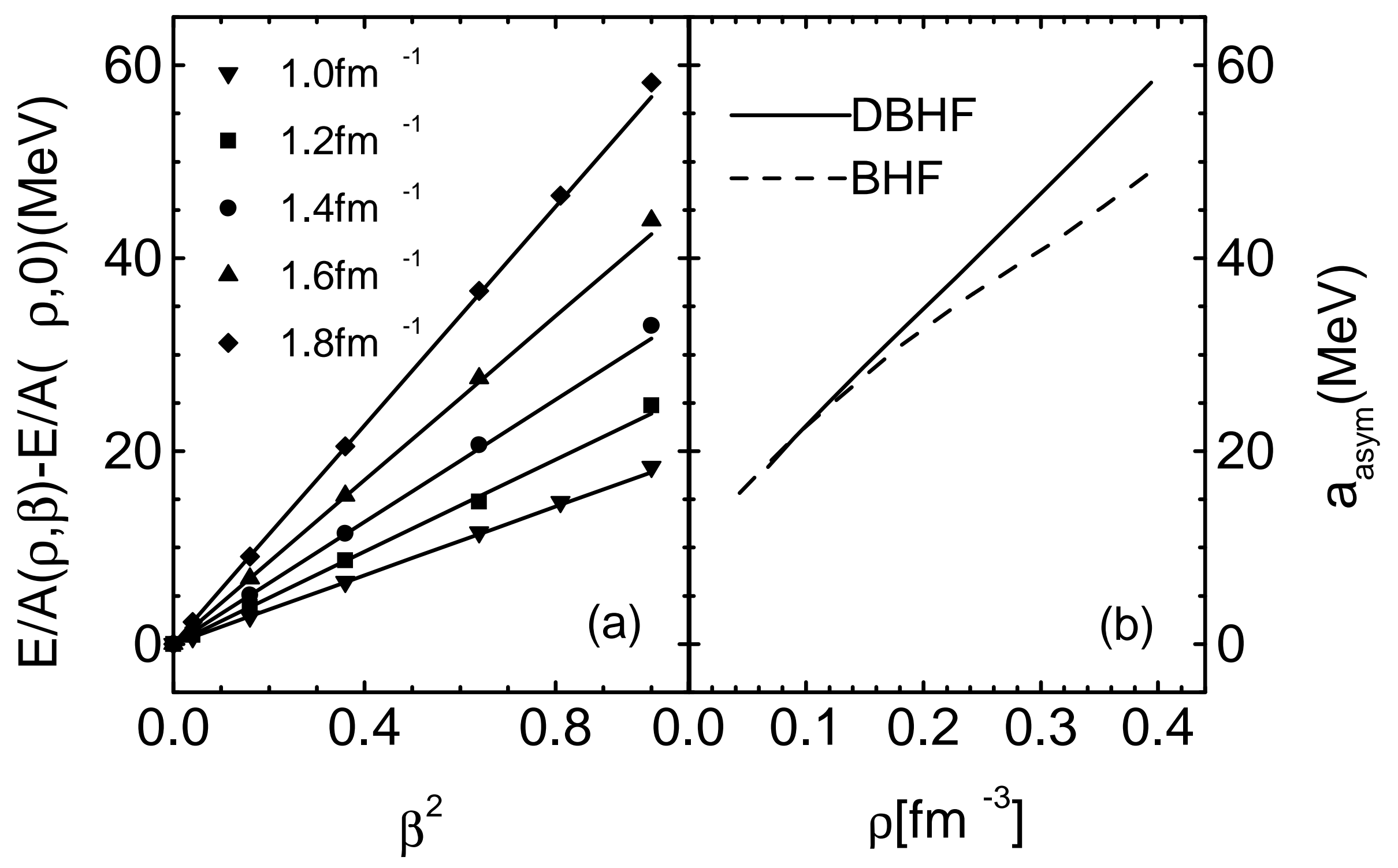




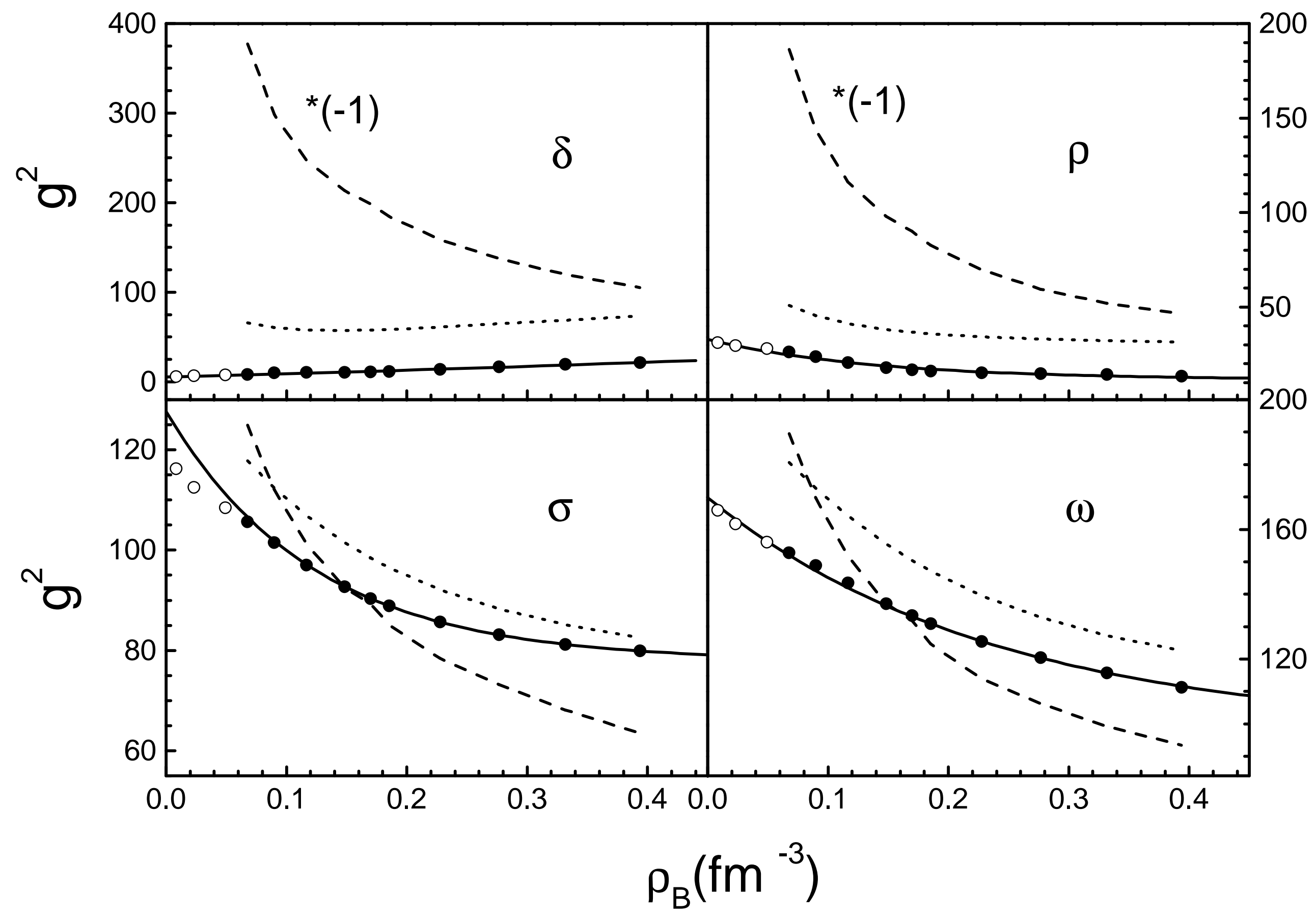



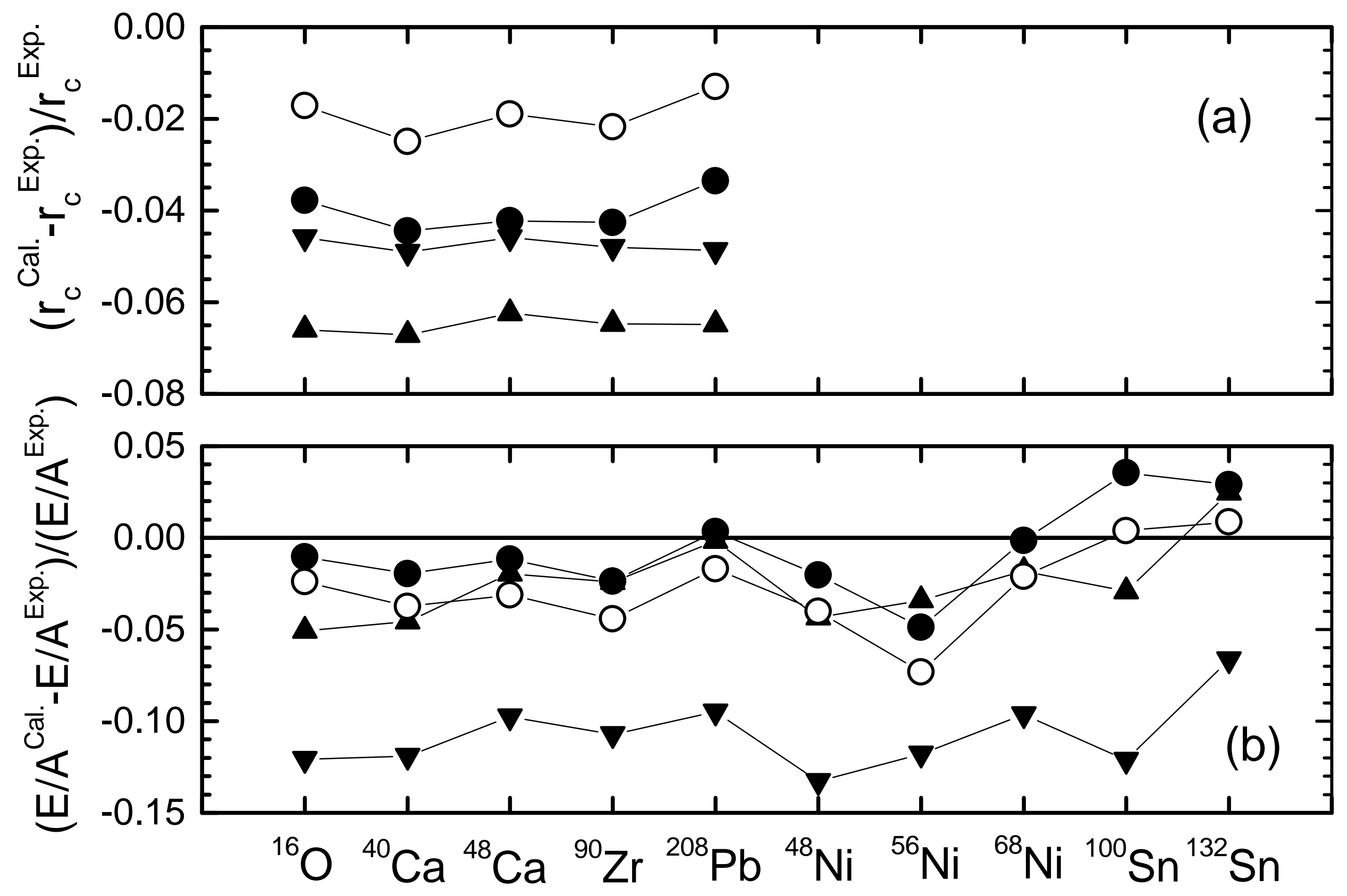


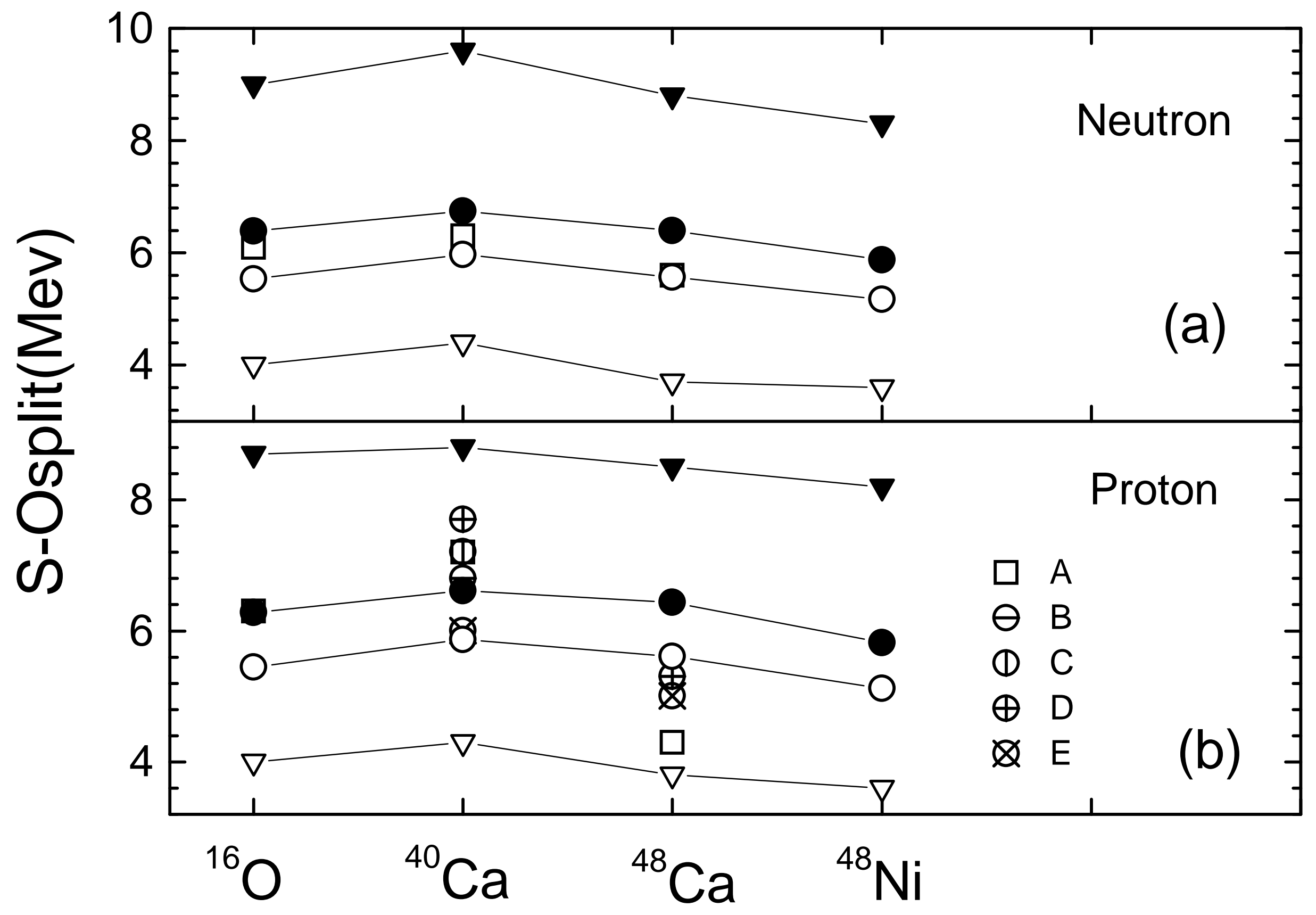

